\title{
Good copper, bad copper
}

Copper has long been known for its antimicrobial properties, which have been linked to the production of toxic hydroxyl radicals and the inhibition of Fe-S-dependent enzymes. However, the fungal pathogen Cryptococcus neoformans is resistant to copper toxicity, and copper has been implicated in C. neoformans virulence. A new study in Cell Host \& Microbe now clarifies how copper levels influence C. neoformans pathogenesis during pulmonary infection.

C. neoformans has a range of copper-responsive genes; some, such as copper transporter 1 (CTR1) and CTR4, enhance copper acquisition and are induced when the copper supply is limited, whereas others, such as the metallothionein genes $C M T 1$ and CMT2, serve as copper detoxifiers and are induced when copper is in excess. However, it was unclear which condition C. neoformans encounters in vivo. Using C. neoformans reporter strains in which the luciferase gene was under the control of either the CTR4 or CMT1 promoter, to allow monitoring of fungal copper-responsive genes in living animals, Ding et al. showed that in mouse lungs, expression from the

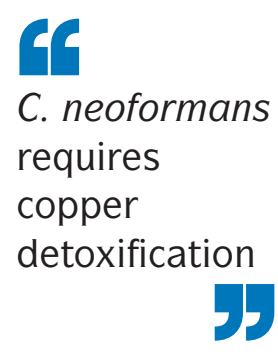

CMT1 promoter rose steadily after infection, whereas expression from the CTR4 promoter remained low. This indicates that $C$. neoformans requires copper detoxification during pulmonary infection. In accordance with this, a mutant strain in which both CMT1 and CMT2 were deleted produced fewer lung colonies and resulted in less mortality in mice than the wild-type fungus. In vitro, the double-mutant strain showed reduced growth in the presence of copper, compared with the wild-type fungus, but normal growth in the presence of other metals. Furthermore, purified

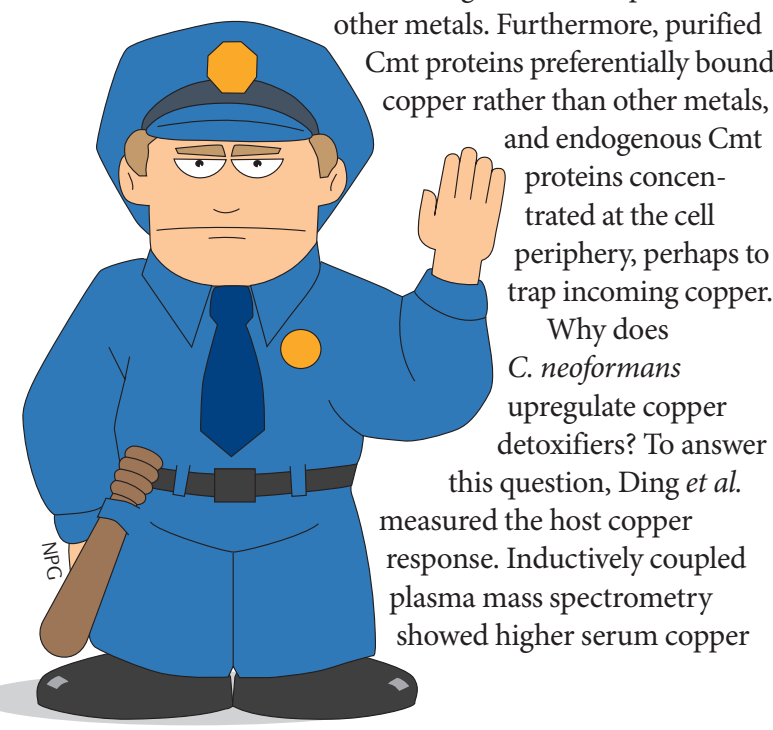

concentrations in mice infected with C. neoformans than in uninfected mice, and immunoblotting revealed that bronchoalveolar lavage cells (which are mostly macrophages) from infected mice produced more of the mouse transporter CTR1 (also known as SLC31A1), which imports copper into the cytoplasm. Usually, macrophages concentrate copper in the phagosome to kill phagocytosed microorganisms. In the macrophages recovered from C. neoformansinfected mice, however, the levels of the phagosomal copper importer ATP7A were decreased. Therefore, the authors conclude that the host mounts an antimicrobial copper response after C. neoformans infection and the pathogen responds by inhibiting copper accumulation in phagosomes. Further work is required to decipher the precise molecular mechanisms involved.

Ursula Hofer

ORIGINAL RESEARCH PAPER Ding, C. et al. Cryptococcus neoformans copper detoxification machinery is critical for fungal virulence. Cell Host Microbe 13, 265-276 (2013)

FURTHER READING Hood, M. I. \& Skaar, E. P.

Nutritional immunity: transition metals at the pathogen-host interface. Nature Rev. Microbiol. 10, 525-537 (2012) 